\title{
ANÁLISE DE PROJEÇÕES DAS MUDANÇAS CLIMÁTICAS SOBRE PRECIPITAÇÃO E TEMPERATURA NAS REGIÕES HIDROGRÁFICAS BRASILEIRAS PARA O SÉCULO XXI
}

Greicy Kelly da Silva (1) Doutoranda em Engenharia Civil com Área de Concentração em Recursos Hídricos, Universidade Federal do Ceará (UFC) Fortaleza (CE), Brasil.

\section{Cleiton da Silva Silveira (1)}

Professor Adjunto, Departamento de Engenharia Hidráulica e Ambiental, UFC - Fortaleza (CE), Brasil.

\section{Marx Vinicius}

\section{Maciel da Silva}

Doutorando em Engenharia Civil com Área de Concentração em Recursos Hídricos, Departamento de Engenharia Hidráulica e Ambiental, UFC - Fortaleza (CE), Brasil.

\section{Antônio Duarte Marcos Júnior (1)}

Mestrando em Ciências Físicas Aplicadas ao Desenvolvimento do Semiárido brasileiro, Universidade Estadual do Ceará (UECE) Fortaleza (CE), Brasil.

\section{Francisco de Assis}

\section{Souza Filho (1)}

Professor Associado, Departamento de Engenharia Hidráulica e Ambiental, UFC Fortaleza (CE), Brasil.

\section{Sullyandro}

\section{Oliveira Guimarães (1)}

Pesquisador, Departamento de Engenharia Hidráulica e Ambiental, UFC - Fortaleza (CE), Brasil.

\section{Endereço para correspondência:} Greicy Kelly da Silva - Departamento de Engenharia Hidráulica e Ambiental, Bloco 713, 10 AndarCampus do Pici-CEP: $60455.760-$ Fortaleza (CE), Brasil - E-mail: greicykellysilvagks@gmail.com

Recebido em: 27/11/2019

Aceito em: 10/03/2020

\section{RESUMO}

A fim de obter informações acerca dos possíveis impactos no regime dos campos de precipitação e no regime de temperatura nas regiões hidrográficas brasileiras em decorrência do aumento das emissões de gases de efeito estufa, este trabalho teve como objetivo analisar as projeções resultantes de nove modelos participantes do Coordinated Regional Climate Downscaling Experiment (CORDEX), considerando os cenários representative concentration pathways (RCP) 4.5 e RCP8.5 para o séculoXXI. Os modelosutilizados foram: Canadian Centre for Climate Modelling and Analysis - Canadian Earth System Model (The second generation) (CCCma-CanESM2), Commonwealth Scientific and Industrial Research Organization (version Mk3-6-0) (CSIRO-Mk3-6-0), Irish Centre for High End Computing - European Community - EARTH (ICHEC-EC-EARTH), Institut Pierre Simon Laplace - 5 Component Models version A - Medium Resolution (IPSL-CMSA-MR), Model for Interdisciplinary Research on Climate version 5 (MIROC5), Hadley Center Global Environment Model version 2 - Earth System (HadGEM2-ES), Max Planck Institute - Meteorology - Earth System Model (MPIM-ESM), Norwegian Climate Centre - Norwegian Earth System Model version 1 - Medium resolution (NCC-NorESM1-M) e National Oceanic and Atmospheric Administration - Geophysical Fluid Dynamics Laboratory - Earth System Model version $2 M$ (NOAA-GFDL-ESM2M). Foram analisadas as anomalias e a tendência dos campos de precipitação e temperatura médias anuais no período de 2006 a 2095. Todos os modelos projetaram aumento da temperatura em todas as regiões. Para o cenário $\mathrm{RCP8.5}$, a anomalia da temperatura indicou aumento de até 1,58 ㅇ $\mathrm{C}$ na região hidrográfica amazônica. A precipitação também pode aumentar em algumas regiões hidrográficas. A mediana das anomalias sugeriu aumentos entre 10 e $30 \%$ no Atlântico Leste, Atlântico Nordeste Ocidental, Atlântico Nordeste Oriental, Paraguai, Parnaíba, Tocantins-Araguaia e São Francisco. Anomalias negativas foram identificadas no sudeste e principalmente no sul do Brasil, indicando reduções na precipitação. O teste de Mann-KendallSen sugeriu uma possível intensificação no regime de precipitações anuais em grande parte das regiões hidrográficas, exceto as do Atlântico Sul, Paraná e Uruguai. Em ambos os cenários, o teste apontou ausência de tendência na região do Atlântico Sul pela maioria dos modelos. Todos os modelos apresentaram tendência positiva significativa para a temperatura nos dois cenários e em todas as regiões. A maior e a menor tendência de aquecimento foram observadas no norte e no sul do país, respectivamente.

Palavras-chave: CSIRO-Mk3-6-0; ICHEC-EC-EARTH; HadGEM2-ES; mudanças climáticas.

\section{ABSTRACT}

In order to obtain information about the possible impacts on the precipitation and temperature fields regime in the Brazilian hydrographic regions, due to 
the increase in greenhouse gas emissions, this study aimed to analyze the projections resulting from nine models participating in the Coordinated Regional Climate Downscaling Experiment (CORDEX), considering the scenarios RCP4.5 and RCP8.5 for the $21^{\text {st }}$ century. The models used were CCCma-CanESM2, CSIRO-Mk3-6-0, ICHEC-EC-EARTH, IPSL-CMSAMR, MIROC5, HadGEM2-ES, MPI-M-ESM, NCC-NorESM1-M, and NOAA-GFDL-ESM2M. The anomalies and trends of the mean annual rainfall and temperature fields in the period from 2006 to 2095 were analyzed. All models projected temperature increases in all regions. For the RCP8.5 scenario, the temperature anomaly indicated an increase of up to $1.58^{\circ} \mathrm{C}$ in the Amazonian hydrographic region. Precipitation is also expected to increase in some hydrographic regions. The median of anomalies suggested increases of between 10 and $30 \%$ in the Eastern Atlantic, Western Northeast Atlantic, Eastern Northeast Atlantic, Paraguay, Parnaiba, Tocantins-Araguaia, and San Francisco. Negative anomalies were identified in the Southeast and mainly in the South of Brazil, indicating reductions in precipitation. The ManKendall-Sen test suggested a possible intensification of the annual rainfall regime in most hydrographic regions, except those in the South Atlantic, Parana, and Uruguay. In both scenarios, the test showed no trend in the South Atlantic region by most models. All models showed a significant positive trend for temperature in both scenarios and in all regions. The highest and lowest warming trends were observed in the North and South of the country, respectively.

Keywords: CSIRO-Mk3-6-0; ICHEC-EC-EARTH; HadGEM2-ES; climate change.

\section{INTRODUÇÃO}

A mudança climática vem instituindo-se como um desafio global diante dos impactos que são produzidos em todas as esferas da existência (ANA, 2016). Um dos principais efeitos decorrentes das mudanças do clima recai sobre a esfera hídrica: alterações significativas no ciclo da água (ANA, 2016; SILVEIRA et al., 2016). De acordo com a Agência Nacional de Águas (ANA, 2016), essas modificações comprometem a disponibilidade hídrica, aumentando a frequência de eventos hidrológicos críticos. Esses problemas afetam, por exemplo, o abastecimento de água potável, o saneamento e a produção de energia e alimentos, sistemas que se relacionam inextricavelmente, caracterizando uma abordagem denominada de nexo água-alimento-energia.

$\mathrm{Na}$ abordagem do nexo, é interessante destacar como a integração entre os sistemas opera em via de mão dupla. No Brasil, o elo entre recursos hídricos e energia sustenta os processos de abastecimento, tratamento e uso da água, ao mesmo passo que fundamenta a geração de hidroeletricidade (ROTHAUSEN; CONWAY, 2011). A interconexão entre os sistemas de energia e de alimentos, por sua vez, norteia a produção de biocombustíveis no país. Além da produção de alimentos, a agricultura brasileira oferta matéria-prima para produzir combustíveis líquidos de diferentes fontes, como a cana-de-açúcar, a soja, o girassol, a mamona, o milho, o sebo bovino, entre outras (ANP, 2017; AZEVEDO; LIMA, 2016). A oferta interna de energia considerando essas fontes caracteriza uma das maiores matrizes de energias renováveis do mundo (AZEVEDO; LIMA, 2016; BRASIL, 2018). A produção de etanol por meio da cana-de-açúcar destaca mundialmente o Brasil como grande produtor de biocombustíveis. Logo, a produção agrícola brasileira desempenha papel fundamental na economia do país, atendendo à demanda de produção dos biocombustíveis e atuando como fonte essencial de renda no cenário econômico nacional (BOULAY et al., 2018; ANA, 2017).

De outra forma, a relação entre alimentos e água subsidia a segurança alimentar, sendo a água um recurso indispensável na produção dos alimentos. O Brasil ocupa o quinto lugar (63.994.479 ha) no mundo em área cultivada, tendo como predecessores a Índia, os Estados Unidos, a China e a Rússia. Sua parcela agrícola correspondia a $3,42 \%$ da área total cultivada no mundo e $7,6 \%$ das terras cultivadas no próprio país no ano de 2016, informações que ressaltam o potencial mundial da agricultura brasileira (MIRANDA, 2018).

Em escala global, o Brasil é uma das regiões mais suscetíveis às mudanças climáticas (VAN VLIET et al., 2013; STOCKER et al., 2013). ANA (2016) e Silveira et al. 
(2014; 2018$)$ indicam que a geração de energia hidroelétrica no país é afetada pelas alterações ocorridas nos padrões de escoamento dos rios e influenciadas principalmente pelos efeitos da mudança do clima sobre os padrões de precipitação em conjunto com modificações no uso e na ocupação do solo. Em resposta, os impactos atingem especialmente o sistema elétrico brasileiro, no qual se tem observado o crescimento da demanda energética, que por sua vez exige a expansão da capacidade instalada por meio da construção de usinas, entre elas hidroelétricas, provocando alterações nos ecossistemas (SOITO; FREITAS, 2011; STOCKER et al., 2013).

De modo a garantir a segurança energética do país, tem-se buscado adotar outras fontes de geração de eletricidade, a citar o uso de usinas termelétricas, no entanto o incremento energético por essa fonte, além de demandar alto custo operacional, eleva a emissão de gases de efeito estufa (GEEs), acentuando o aquecimento global (MERCURE et al., 2019; PRADO JÚNIOR et al., 2016). Nesse contexto, o celeiro energético pautado no uso dos biocombustíveis destaca-se por ter emissão cerca de duas vezes menor de GEEs quando comparada à quantidade emitida pela queima de combustíveis fósseis (BERGER et al., 2015), entretanto a produção de biocombustíveis requer alto consumo hídrico e provoca mudanças no uso da terra motivadas pela necessidade de expandir a área ocupada para fins de produção, bem como suprir a demanda por culturas para exportação, o que implica a competição com a produção de alimentos e o aumento do desmatamento (GOPALAKRISHNAN et al., 2009; MERCURE et al., 2019). Em suma, os impactos das mudanças climáticas são sentidos em todos os sistemas integrados pelo nexo água-alimento-energia.

Estudos voltados para identificar e avaliar como as alterações e a variabilidade do clima podem afetar o globo atuam como subsídios fundamentais na elaboração de planos mitigadores aos impactos gerados (SILVEIRA et al., 2018). Esses estudos requerem informações de escala regional/local que podem ser obtidas de análises provenientes de modelos climáticos globais (MCGs) com a aplicação de técnicas tais como o downscaling dinâmico, que regionaliza a escala desses dados (SALES et al., 2015). Nesse sentido, surgiu o Projeto Coordinated Regional Climate Downscaling Experiment (CORDEX), que fornece uma metodologia padronizada dos experimentos de downscaling dinâmico sobre alguns domínios, em escala regional, das projeções globais provindas dos modelos que compõem o Coupled Model Intercomparison Project Phase 5 (CMIP5) (GIORGl; JONES; ASRAR, 2009; GUIMARÃES et al., 2016).

Alguns estudos recentes vêm sendo realizados com o downscaling dinâmico abordando a metodologia do CORDEX sobre domínios que envolvem o Brasil e a América do Sul. Guimarães et al. (2016) e Sales et al. (2015) investigaram o clima atual e futuros cenários climáticos utilizando diferentes modelos climáticos regionais (MCRs) para o Nordeste do Brasil (NEB) e regiões norte e sul do NEB, respectivamente. Da mesma forma, Reboita et al. (2014) determinaram a melhor configuração do MCR Regional Climate Model version 4.3 (RegCM4.3) para simular o clima no domínio da América do Sul para uso no CORDEX. Ainda em relação a esse domínio, três MCGs (Max Planck Institute - MPI, Geophysical Fluid Dynamics Laboratory - GFDL e Hadley Center Global Environment Model - HadGEM2) foram usados para o downscaling do MCR RegCM4 no trabalho de Da Rocha et al. (2014), com o intuito de investigar o sinal de precipitação do fenômeno El Niño Oscilação Sul (ENSO) na América do Sul em três períodos - presente (1975-2005), próximo ao futuro (2020-2050) e futuro distante (2070-2098) -, em dois cenários de GEEs: representative concentration pathways (RCP) 4.5 e RCP8.5.

O objetivo deste trabalho foi analisar as projeções de alguns modelos participantes do CORDEX considerando os cenários RCP4.5 e RCP8.5 para o século $X X I$, a fim de identificar mudanças nos padrões de variabilidade na precipitação e temperatura nas regiões hidrográficas do Brasil.

\section{MATERIAIS E MÉTODOS}

\section{Região de estudo}

A área de estudo compreende as 12 regiões hidrográficas (RHs) brasileiras: Amazônica, Atlântico Leste, Atlân- tico Nordeste Ocidental, Atlântico Nordeste Oriental, Atlântico Sudeste, Atlântico Sul, Paraguai, Paraná, 
Parnaíba, São Francisco, Tocantins-Araguaia e Uruguai, conforme mostra a Figura 1. A seguir, a conjuntura dos recursos hídricos no Brasil elaborada pela ANA (2015) descreve essas regiões.

Inserida na Bacia Amazônica, a RH Amazônica possui uma área que totaliza aproximadamente $45 \%$ do território brasileiro e abrange os estados do Acre, do Amazonas, de Rondônia, de Roraima, do Amapá, do Pará e de Mato Grosso. Apresentando grande disponibilidade hídrica superficial (o equivalente a $81 \%$ da disponibilidade superficial do Brasil) e compondo uma expressiva rede de drenagem, possui como rios de destaque: Purus, Juruá, Xingu, Solimões, Madeira, Negro e Guaporé. Sua precipitação média anual é de $2.205 \mathrm{~mm}$, valor que representa cerca de $25 \%$ a mais do que a média nacional $(1.761 \mathrm{~mm})$.

A RH Atlântico Leste, a RH Atlântico Nordeste Oriental, a RH do Parnaíba e a RH do São Francisco compreendem uma área percentual do território brasileiro de pouco mais de 3,9, 3,4, 3,9 e 7,5\% respectivamente. Uma grande parte de todas essas regiões hidrográficas situa-se no semiárido nordestino, sendo caracterizada pelos períodos prolongados de estiagem, motivada pela baixa pluviosidade e alta evapotranspiração, o que Ihe confere precipitação média anual inferior à média nacional. A RH Atlântico Leste distribui-se pelos estados da Bahia (69\%), de Minas Gerais (26\%), de Sergipe (4\%) e do Espírito Santo (1\%), contendo em seu leque de rios principais o Salgado e Gavião, enquanto a Atlântico Nordeste Oriental abrange seis estados: Piauí, Ceará, Rio Grande do Norte, Paraíba, Pernambuco e Alagoas. Nessa região, os principais açudes são banhados pelos rios Jaguaribe e Piranhas-Açu. A RH Parnaíba, por sua vez, abrange porções dos estados do Piauí (77\%), do Maranhão (19\%) e do Ceará (4\%). Atravessando diferentes biomas (cerrado, caatinga e costeiro), entre os seus principais cursos d'água se destacam os rios Parnaíba, Piauí e Poti. De outra forma, a $\mathrm{RH}$ do São Francisco compreende sete estados: Bahia, Minas Gerais, Pernambuco, Alagoas, Sergipe, Goiás e Distrito Federal. Fomentando a base de suprimento de energia da Região Nordeste, essa RH possui $10.708 \mathrm{MW}$ de potencial hidroelétrico instalado, o que equivale a $12 \%$ do

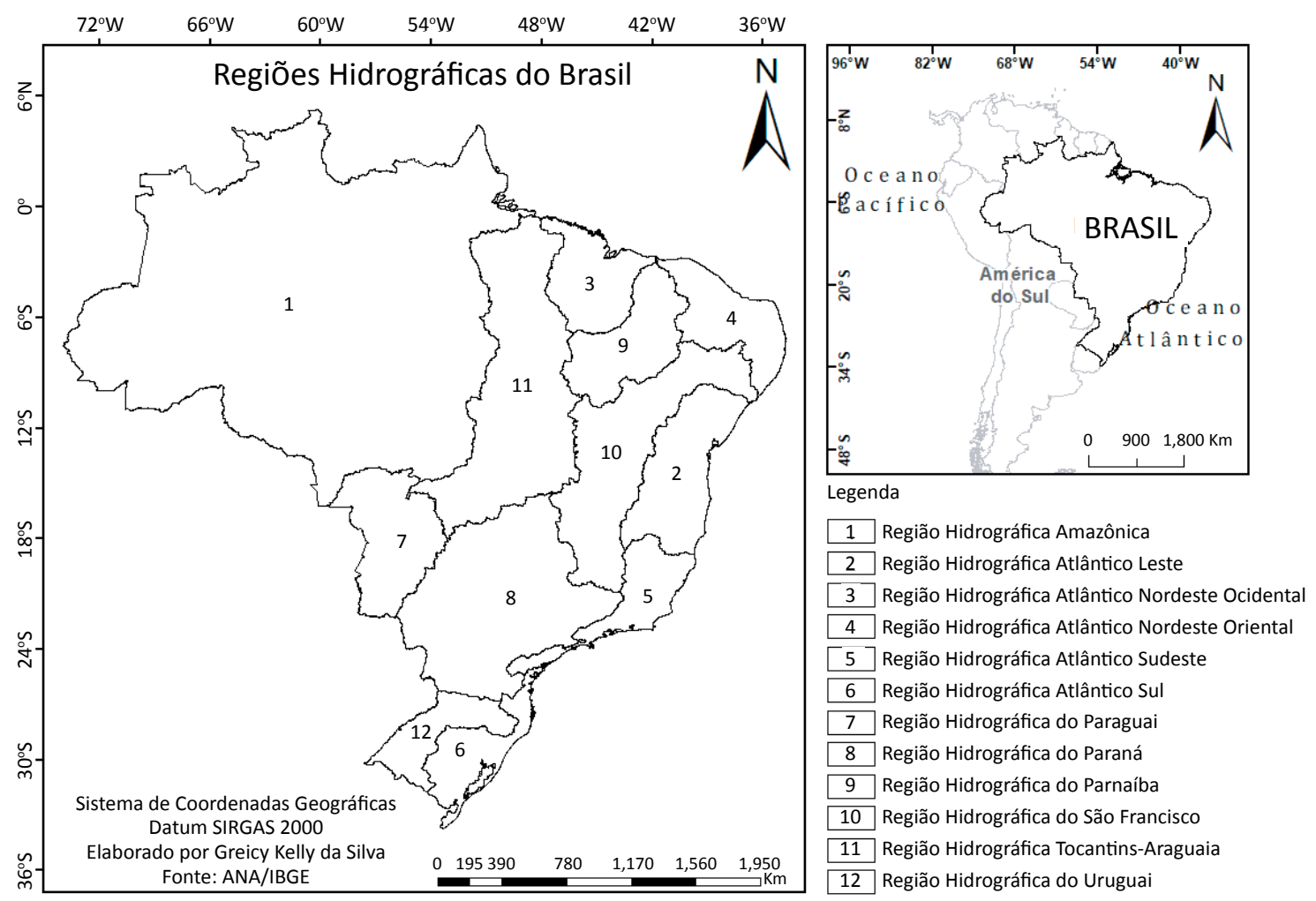

Figura 1 - Regiões hidrográficas do Brasil. 
total no país, destacando-se as usinas de Xingó, Paulo Afonso IV, Luiz Gonzaga e Sobradinho.

A RH Atlântico Nordeste Ocidental abrange 3\% do território nacional, compreendendo o estado do Maranhão e pequena parcela do Pará. Seus rios principais são o Gurupi, o Mearim, o Itapecuru e o Munim. Sua precipitação média anual é um pouco abaixo da média do país: 1.700 mm. A RH Atlântico Sudeste, a RH Atlântico Sul e a RH do Paraná são consideradas as regiões hidrográficas mais expressivas do território brasileiro em termos de contingente populacional e desenvolvimento econômico. Enquanto a Atlântico Sul possui, em área, um total percentual equivalente a 2,2\% do país (envolvendo quatro estados - São Paulo, Paraná, Santa Catarina e Rio Grande do Sul), a Atlântico Sudeste detém 2,5\%, alcançando cinco unidades da Federação (Minas Gerais, Espírito Santo, Rio de Janeiro, São Paulo e Paraná), e a do Paraná, com área equivalente a cerca de $10 \%$ do território nacional, abrange sete estados: São Paulo, Paraná, Mato Grosso do Sul, Minas Gerais, Goiás, Santa Catarina e Distrito Federal. A RH do Paraná é ainda a responsável pela maior demanda de recursos hídricos do Brasil. Seu potencial hidroelétrico aproveitado corresponde a $47,5 \%$ do total instalado no país, e ela apresenta o maior aproveitamento do potencial hidráulico disponível. Itaipu, Ilha Solteira e Furnas destacam-se entre as inúmeras usinas hidroelétricas em operação nessa região.

\section{Dados observacionais}

A base de dados observacionais utilizada para verificar a destreza dos modelos do CORDEX sobre as 12 regiões hidrográficas é proveniente do Global Precipitation Climatology Centre (GPCC) e fornecida pelo Principal Science Advisor National Oceanic and Atmospheric Administration (PSA NOAA), por Oceanic and Atmospheric Research (OAR) e por Earth System Research Laboratory (ESRL) (NEW; HULME; JONES, 1999), conforme o link http://

\section{Projeto CORDEX}

Este trabalho utilizou dados mensais de campos de precipitação (variável pr) e temperatura do ar próximo à superfície (variável tas - temperatura média) obtidos de nove modelos climáticos globais (ver Tabela 1) que atuaram como condição de contorno no processo de downscaling dinâmico para o modelo regional sueco Rossby Centre regional atmospheric model (RCA4) pelo
Também denominada de Bacia do Alto Paraguai, a RH do Paraguai possui área de $363.446 \mathrm{~km}^{2}$ (4,3\% do território brasileiro) e abrange parte dos estados do Mato Grosso e do Mato Grosso do Sul. Essa região situa a maior área úmida contínua do mundo: o pantanal mato-grossense (no qual $90 \%$ de sua extensão está localizada no Brasil). Entre seus principais cursos d'água, destacam-se o Rio Paraguai. Com rico potencial hidroelétrico, a região possui $1,4 \mathrm{GW}$ já aproveitado, o que corresponde a $1,3 \%$ do total instalado no Brasil. Também com importância significativa para o país, a RH do Uruguai sobressai em função das suas atividades agroindustriais e do potencial hidroelétrico que se distribui ao longo do Rio Uruguai e de seus afluentes, gerando potência instalada de aproximadamente $6.000 \mathrm{MW}$. Com, em território nacional, área equivalente a $3 \%$, abrange partes dos estados do Rio Grande do Sul (74\%) e Santa Catarina (26\%). É frequente, no entanto, a ocorrência de eventos críticos nessa região, tais como enchentes, alagamentos, enxurradas e inundações.

À frente da expansão da fronteira agrícola, a RH Tocantins-Araguaia destaca-se pelo cultivo de grãos e pelo potencial hidroenergético. Com uma área que engloba $10,8 \%$ do território brasileiro, a região abrange os estados de Goiás (21\%), Tocantins (30\%), Pará (30\%), Maranhão (4\%), Mato Grosso (15\%) e o Distrito Federal $(0,1 \%)$. Detém $15 \%$ da capacidade da hidroeletricidade total instalada no país, com potencial de 13,14 GW.

www.esrl.noaa.gov/psd/data/gridded/data.gpcc.html. $\mathrm{O}$ conjunto de dados usado corresponde à climatologia de precipitação de 1901 a 2013, em uma grade regular com resolução espacial de 0,5 . Esse banco de dados foi gerado por pesquisadores do GPCC que reconstruíram dados observacionais de precipitação mensal do globo para a grade de espaçamento de $0,5 \times 0,5 \circ$, com base em métodos estatísticos diversos (SCHNEIDER et al., 2011).

CORDEX, obtendo simulações mais refinadas e com resolução espacial de $0,44 \times 0,44$ o. As simulações tiveram como domínio a área da América do Sul (SAM-44), e foram calculadas as médias espaciais dos resultados para cada ponto de grade, sendo esta obtida por meio da interpolação bilinear da grade original de SAM-44 sobre cada região hidrográfica do Brasil. 
Tabela 1 - Modelos globais do Coordinated Regional Climate Downscaling Experiment (CORDEX) utilizados e suas respectivas instituições/agências e países.

\begin{tabular}{|l|r|}
\hline Modelos & $\begin{array}{r}\text { Instituição ou Agência; País } \\
\hline \text { CCCma-CanESM2 }\end{array}$ \\
\hline CSIRO-Mk3-6-0 & $\begin{array}{r}\text { Canadian Centre for Climate Modelling and Analysis; Canadá } \\
\text { Commonwealth Scientific and Industrial Research Organization em } \\
\text { colaborão com Queensland Climate Change Centre of Excellence; Austrália }\end{array}$ \\
\hline ICHEC-EC-EARTH & Irish Centre for High-End Computing; Irlanda \\
\hline IPSL-CM5A-MR & Institut Pierre Simon Laplace; Paris \\
\hline MIROC5 & Environmental Studies e Japan Agency for Marine-Earth Science and Technology; Japão \\
\hline HadGEM2-ES & Atmosphere and Ocean Research Institute, National Institute for \\
\hline MPI-M-ESM & Max Planck Institute for Meteorology; Alemanha \\
\hline NCC-NorESM1-M & $\begin{array}{r}\text { Madley Centre; Reino Unido } \\
\hline \text { NOAA-GFDL-ESM2M }\end{array}$ \\
\hline
\end{tabular}

A base de dados compreende as séries mensais de 1951 a 2005 (período de referência), e de 2006 a 2100 foi o período considerado para as projeções. Estas abrangeram o cenário intermediário RCP4.5 e o cenário de emissões muito altas de GEEs, o RCP8.5. Vale ressaltar, de acordo com o quinto relatório do Painel Intergovernamental sobre Mudanças Climáticas (IPCC), que as RCPs são utilizadas para elaborar as projeções descrevendo quatro diferentes caminhos do século XXI (RCP2.6: cenário otimista; RCP4.5 e RCP6.0: cenários intermediários; e RCP 8.5: cenário mais pessimista), considerando as emissões de GEEs, as concentrações atmosféricas, as emissões de poluentes do ar e o uso do solo (STOCKER et al., 2013). Ainda nesse contexto, as forçantes radiativas, que definem o nome das RCPs, sugerem estabilização ou pico no fim do século em questão, pressupondo, portanto, níveis de 4,5 e $8,5 \mathrm{~W} \cdot \mathrm{m}^{-2}$ para os cenários abordados neste trabalho.

\section{Análise de tendência e variabilidade das séries temporais observadas e modeladas}

Esta seção apresenta as metodologias utilizadas para analisar as projeções dos campos de precipitação e temperatura dos modelos globais do CORDEX, bem como as tendências e os padrões de variação das séries his-

\section{Análise das projeções}

As projeções de precipitação e temperatura dos modelos globais do CORDEX foram analisadas nas 12 regiões

\section{Cálculo das anomalias médias anuais}

Neste trabalho, para o cálculo da anomalia média anual $\left(\mathrm{A}_{\text {anual }}\right)$ da precipitação, foi utilizada a Equação 1:

$A_{\text {anual }}=\frac{\left(P_{X X I}^{a}-P_{X X}^{a}\right)}{P_{X X}^{a}} \cdot 100$ tóricas. Quanto à avaliação de tendência/variabilidade, abordou-se o uso de métodos clássicos, como a média e a mediana móvel, o teste de Mann-Kendall e o teste de declividade de Sen.

hidrográficas para o período de 2006 a 2095 para os cenários RCP4.5 e RCP8.5.

Em que:

$P_{x X I}^{a}=$ a média da precipitação anual para o cenário do século XXI;

$P_{x x}^{a}=$ a média da precipitação anual para o cenário histórico. 
De outro modo, para o cálculo da anomalia da temperatura, considerou-se a diferença entre a média da tempe-

\section{Métodos clássicos}

A média $(\mu)$ descreve a amostra que compõe a série como um único valor que representa o centro da distribuição dos dados. O grau de dispersão desses dados é determinado pelo cálculo do desvio padrão $(\sigma)$ (SPIEGEL, 1978). A mediana, por sua vez, atua como uma medida de posição e localiza-se no centro da série, dividindo-a com uma mesma quantidade de elementos antes e depois dessa medida (CORREA, 2003).

Neste estudo, a análise da tendência foi realizada por meio do método de Mann-Kendall. Segundo Moreira e Naghettini (2016), o teste não paramétrico de Mann-Kendall vem sendo amplamente usado para a detecção de tendências em séries de observações hidrológicas, apresentando resultados satisfatórios. Sendo assim, de acordo com Yue, Pilon e Cavadias (2002) e Wagesho, Goel e Jain (2012), para uma série $\left(x_{1}, x_{2}, \ldots, x_{n}\right)$ proveniente de uma amostra de $n$ variáveis aleatórias independentes e identicamente distribuídas, a estatística do teste de Mann-Kendall é dada pela Equação 2:

$S=\sum_{i=1}^{n-1} \sum_{j=i+1}^{n} \operatorname{Sinal}\left(x_{j}-x_{i}\right)$

Em que:

$x_{i}$ e $x_{j}=$ valores sequenciais;

$i$ e $j=$ índices de tempo;

$n=0$ número de elementos da série (MOREIRA; NAGHETTINI, 2016).

O termo $\operatorname{Sinal}\left(x_{j}-x_{i}\right)$ é resultado da Equação 3:

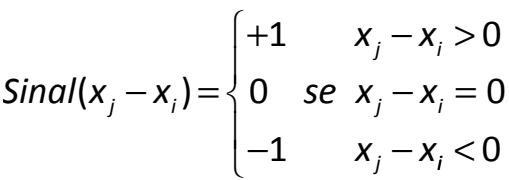

De acordo com Burn e Elnur (2002), na análise de tendência por intermédio do teste de Mann-Kendall, há dois importantes parâmetros a ser considerados: ratura do período no século XXI e a média do período no século XX.

o nível de significância $\alpha$ e a declividade $\beta$, sendo este último determinado pela Equação 4:

$\beta=$ Mediana $\left[\frac{\left(x-x_{i}\right)}{(j-i)}\right]$,

Em que:

$i<j$.

O teste de declividade de Sen, por sua vez, pode ser considerado o complemento do teste de Mann-Kendall, pois fornece a magnitude das tendências detectadas. Segundo Tao et al. (2014), a declividade é estimada pela estatística $Q$, dada pela Equação 5:

$Q_{i j}=\frac{\left(x_{j}-x_{i}\right)}{(j-i)}$,

Nessa situação, $x_{i}$ e $x_{j}$ estão relacionados com os valores da variável em estudo nos tempos $i$ e $j$ e $i<j$ (MOREIRA; NAGHETTINI, 2016). O valor positivo ou negativo para $Q$ indica tendência crescente ou decrescente, respectivamente. A declividade de Sen é dada pela mediana $\operatorname{dos} N$ valores de $Q_{i j}$. No caso de haver apenas uma referência em cada período de tempo e sendo $n$ o tamanho da série, tem-se a Equação 6:

$N=\frac{n(n-1)}{2}$

Para $N$ ímpar, a declividade de Sen é expressa pela Equação 7:

$Q_{\text {mediana }}=Q_{\frac{N+1}{2}}$

Em caso de $N$ par, a declividade de Sen é dada pela Equação 8:

$Q_{\text {mediana }}=\frac{\left[\frac{Q_{\frac{N}{2}}+Q_{\frac{N+2}{2}}}{2}\right]}{2}$

\section{RESULTADOS E DISCUSSÃO}

Na Figura 2 é possível observar precipitações consideradas como discrepantes (outliers), principalmente nas RHs Atlântico Sul e do São Francisco, além do Atlântico
Leste e Parnaíba. No Atlântico Sul, o evento mais atípico aproximou-se dos $1.319 \mathrm{~mm}$. O Atlântico Nordeste Oriental apresentou o menor quartil 1 (inferior) e o 
menor valor de mediana, indicando que $25 \%$ dos seus dados de precipitação estavam abaixo dos $44,17 \mathrm{~mm}$ e que $50 \%$ desses dados foram representados por uma precipitação de 163,03 $\mathrm{mm}$.

De outra forma, a RH Amazônica deteve o maior quartil 3 (superior) da série, concentrando $25 \%$ dos seus dados acima dos $1.432,5 \mathrm{~mm}$, além de denotar a maior mediana, apontando representação da sua precipitação em $1.902,8 \mathrm{~mm}$ no percentual de $50 \%$ dos seus dados. Em contrapartida, foi o Atlântico Nordeste Ocidental que exibiu, entre as demais regiões, maior precipitação acumulada: 1.966,2 $\mathrm{mm}$. Também se verificou a existência de parte da variabilidade interanual em cada região. As regiões hidrográficas com maior variabilidade são as RHs do Atlântico Nordeste Ocidental e Oriental. Nas demais regiões, especialmente as do sul do país, não foi constatada tanta variação, o que talvez demonstre estabilidade no clima dessas regiões. Nas bacias do nordeste, a variação entre anos muito úmidos (com valores próximos a $2.000 \mathrm{~mm}$ no Nordeste Ocidental, por exemplo) e muitos secos impacta consideravelmente na gestão dos recursos hídricos.
De acordo com a Figura 3, a maioria dos modelos do CORDEX divergiu quanto à quantidade de precipitação vista pelo GPCC nas regiões hidrográficas brasileiras. De modo geral, foi na região do Atlântico Sudeste que a maioria dos modelos melhor representou a climatologia observada, superestimando, no entanto, a precipitação nos meses de janeiro, fevereiro, março e abril. As maiores divergências entre os modelos no que tange à quantidade precipitada foram notadas nas regiões Amazônica, Atlântico Leste, Atlântico Nordeste Oriental, Paraguai, Parnaíba, São Francisco e Tocantins-Araguaia, ou seja, em parte do norte e nordeste do Brasil, em que os modelos não conseguiram representar a sazonalidade, principalmente nos meses de novembro a abril, que, em suma, se associam ao período característico da ocorrência de chuvas nessas regiões. De outra forma, uma sazonalidade mais comportada, porém abaixo da climatologia averiguada, foi apontada no sul e sudeste do país, nas RHs Atlântico Sul, Paraná e Uruguai. O modelo CSIRO-Mk3 foi o que mais representou satisfatoriamente a precipitação observada, entretanto subestimou-a na maioria das regiões estudadas.

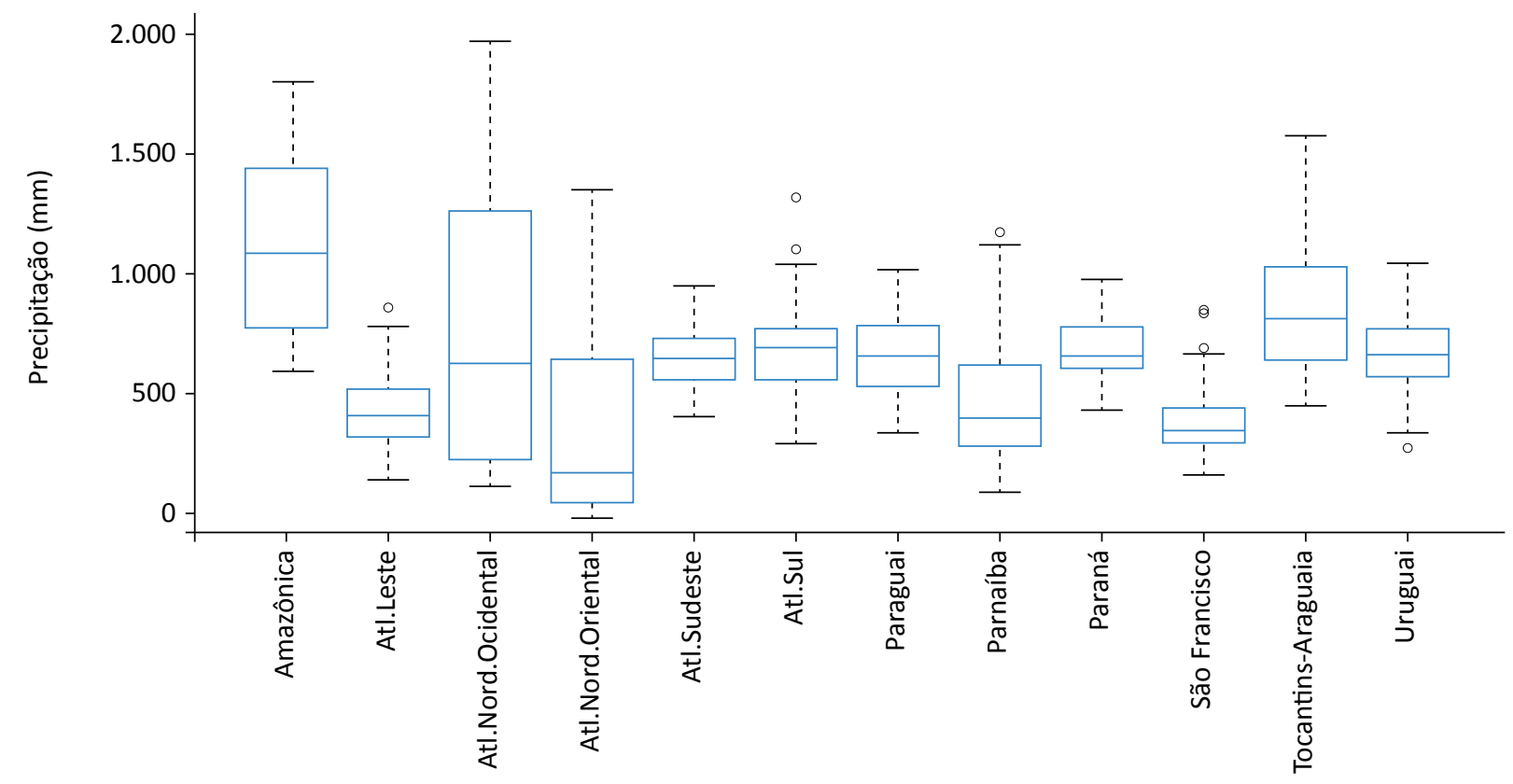

Atl. Nord. Ocid.: Atlântico Nordeste Ocidental; Atl. Nord. Orie.: Atlântico Nordeste Oriental; Atl.: Atlântico.

Figura 2 - Gráfico boxplot da precipitação média anual considerando a série de dados do Global Precipitation Climatology Centre (GPCC) para o período de 1901 a 2013 em todas as regiões hidrográficas estudadas. 

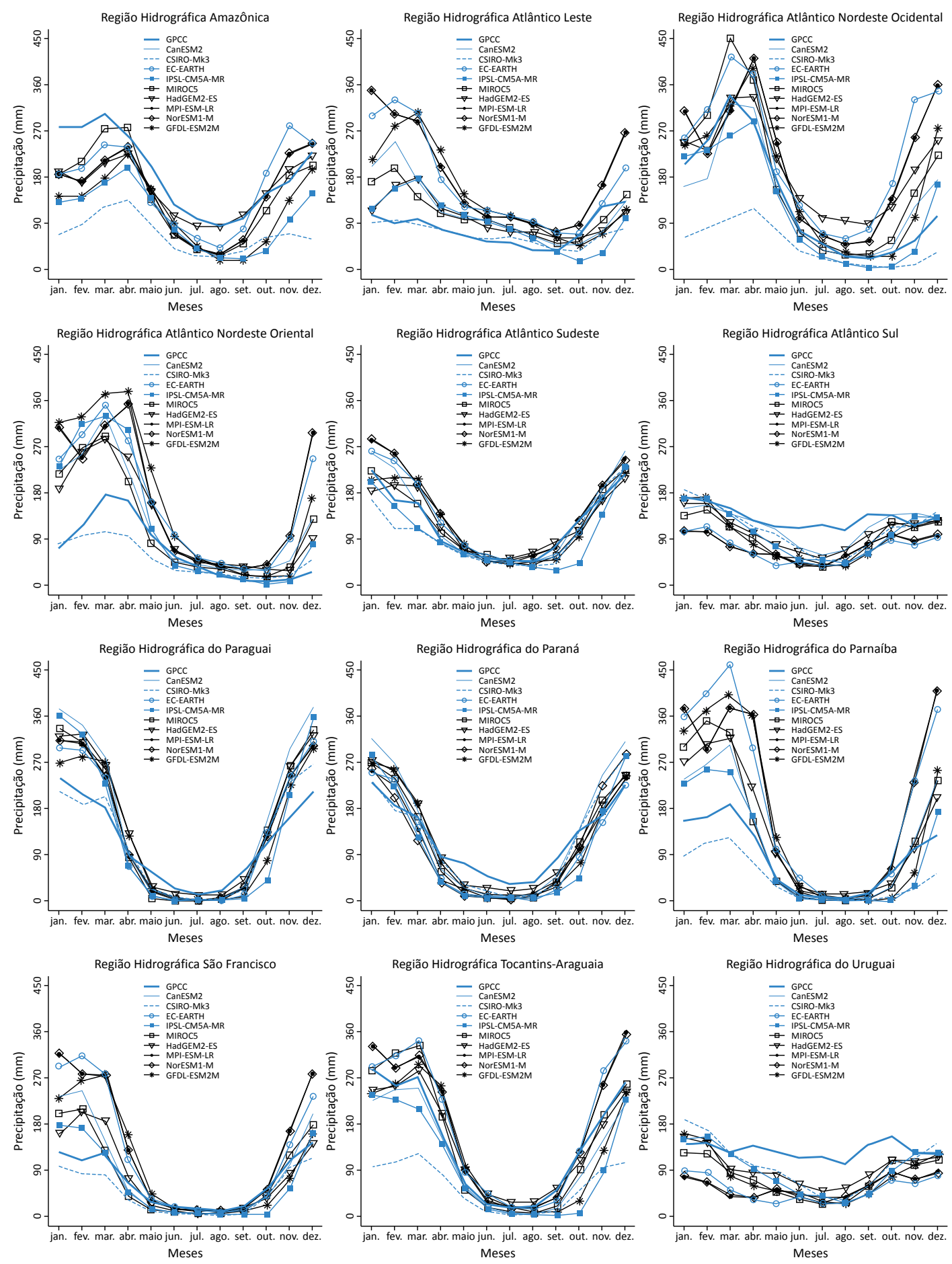

GPCC: Global Precipitation Climatology Centre; CanESM2: Canadian Earth System Model (The second generation); CSIRO-Mk3-6-0: Commonwealth Scientific and Industrial Research Organization (version Mk3-6-0); EC-EARTH: European Community - EARTH; IPSL-CM5A-MR: Institut Pierre Simon Laplace - 5 Component Models version A - Medium Resolution; MIROC5: Model for Interdisciplinary Research on Climate version 5; HadGEM2-ES: Hadley Center Global Environment Model version 2 - Earth System; MPI-ESM-LR: Max Planck Institute - Meteorology - Earth System Model Low Resolution; NorESM1-M: Norwegian Earth System Model version 1 - Medium resolution; GFDL-ESM2M: Geophysical Fluid Dynamics Laboratory - Earth System Model version 2M; Atl.: Atlântico; Atl. Nord.: Atlântico Nordeste.

Figura 3 - Precipitação climatológica dos modelos do Coordinated Regional Climate Downscaling Experiment (CORDEX) e Global Precipitation Climatology Centre (GPCC) para as 12 regiões hidrográficas do Brasil. 


\section{Cenários de precipitação e temperatura}

As Figuras 4A, 4B e 4C mostram a mediana das anomalias de temperatura para o cenário RCP4.5 para os períodos de 2006 a 2035, 2036 a 2065 e de 2066 a 2095, respectivamente. Considerando o cenário RCP8.5, as Figuras 4D, 4E e 4F trazem também para os períodos supracitados, respectivamente, a mediana das anomalias de temperatura. Nas Figuras 4A e 4D é possível observar que, para o primeiro período (2006-2035), a mediana das anomalias de temperatura permaneceu abaixo dos $0,5 \circ \mathrm{C}$ em ambos os cenários (RCP4.5 e RCP8.5). No segundo período (2036-2065) e para o cenário RCP4.5, a mediana das anomalias de temperatura variou entre 0,51 e $0,69{ }^{\circ} \mathrm{C}$ em todas as regiões, de acordo com a Figura 4B. Por outro lado, a Figura 4E ilustra que algumas regiões hidrográficas destacaram no cenário RCP8.5 anomalias superiores, com valores entre 0,8 e 0,89 ․ C. No terceiro período (2066-2095) e sob o cenário otimista, o sinal da anomalia intensificou-se levemente, apresentando a mediana entre 0,70 e $0,81^{\circ} \mathrm{C}$ para a maioria das regiões, excetuando-se as
RHs do São Francisco, Atlântico Leste, Atlântico Sudeste e Atlântico Sul, como pode ser visto na Figura 4C.

Para o mesmo período e cenário RCP8.5, observaram-se as maiores anomalias de temperatura. A Figura 4F mostra que as RHs Amazônica e do Paraguai apresentaram valores de medianas de 1,58 e 1,57 으, respectivamente. Aqui, as regiões Atlântico Leste, Atlântico Sudeste e Atlântico Sul indicaram anomalias também intensas se comparadas a de outros períodos, permanecendo entre 1,13 e 1,18 ㅇ. $C$. A mediana das anomalias das demais regiões variou entre 1,28 e $1,49 \circ C$.

As Figuras 5A, 5B e 5C apresentam a mediana das anomalias de precipitação para o cenário RCP4.5 para os períodos de 2006 a 2035, 2036 a 2065 e de 2066 a 2095, respectivamente. Por outro lado, as Figuras 5D, 5E e 5F mostram também para os períodos mencionados acima, respectivamente, a mediana das anomalias de precipitação no cenário RCP8.5. As Figuras 5A e 5D exibem a mediana da anomalia de precipitação para o primeiro período e
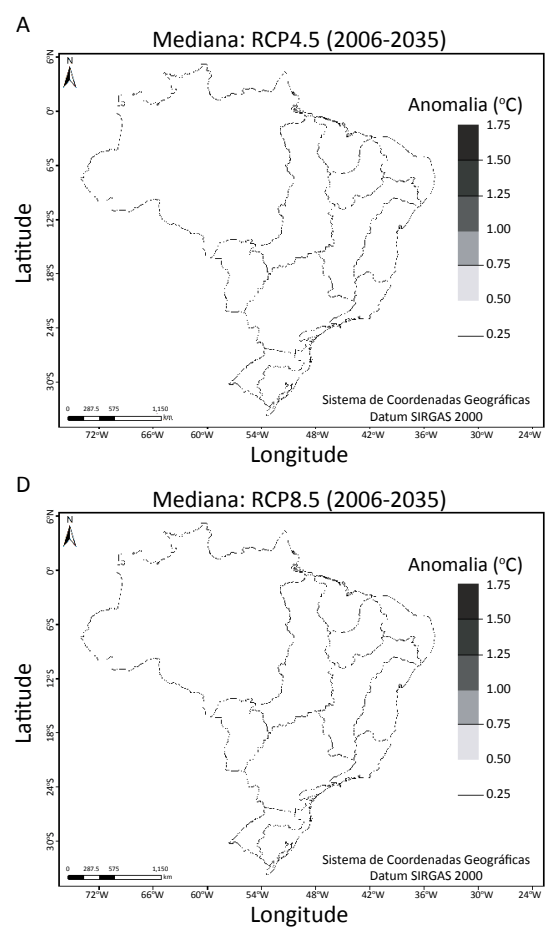

B
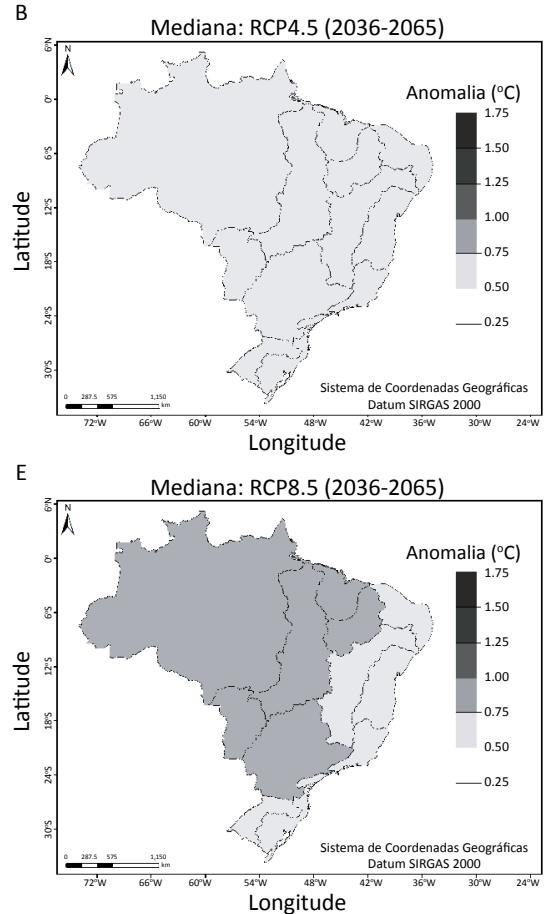

C

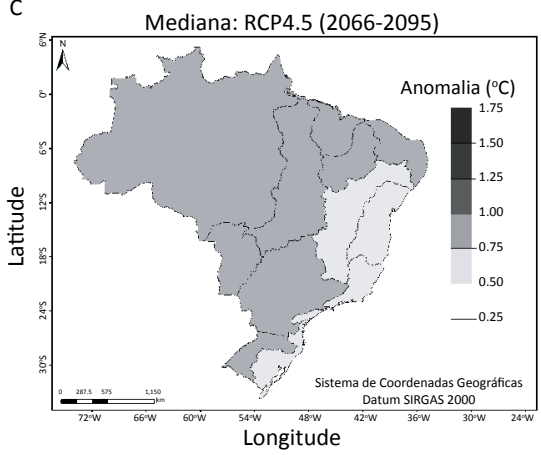

$\mathrm{F}$

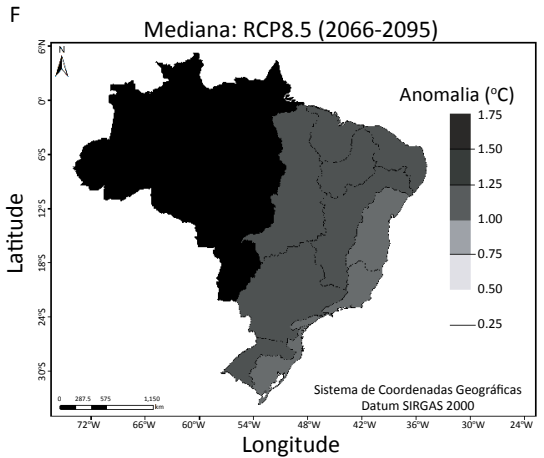

$\mathrm{RCP}$ : representative concentration pathways.

Figura 4 - Mediana das anomalias de temperaturas anuais dos modelos do Coordinated Regional Climate Downscaling Experiment (CORDEX) para o cenário RCP4.5 no período de (A) 2006 a 2035; (B) 2036 a 2065; (C) 2066 a 2095; e para o cenário RCP8.5 no período de (D) 2006 a 2035; (E) 2036 a 2065; (F) 2066 a 2095. 
para os dois cenários como positiva e com módulo inferior a $10 \%$ em todas as regiões hidrográficas. No segundo e no terceiro período, para o cenário RCP4.5 (Figuras 5B e $5 C$ ), houve leve intensificação dos valores de anomalia, variando sua mediana entre 10 e $20 \%$ para as RHs do Tocantins-Araguaia, São Francisco, Parnaíba, Atlântico Nordeste Oriental e Atlântico Nordeste Ocidental. Com exceção desta última e para as demais regiões citadas, sob o cenário RCP8.5 e no segundo período (Figura 5E), destacou-se a mediana da anomalia de precipitação também entre 10 e $20 \%$. De outra forma, ainda sob o cenário pessimista e para o último período projetado, a Figura 5F mostra que as RHs Tocantins-Araguaia, São Francisco, Parnaíba e Atlântico Nordeste Oriental tiveram suas anomalias intensificadas com valores de mediana entre 20 e $30 \%$ e entre 10 e $20 \%$ para as regiões do Atlântico Leste e Paraguai. A RH Atlântico Nordeste Ocidental apresentou-se com as medianas das anomalias mais intensificadas entre as demais no segundo e no terceiro período $-22,3$ e $30,4 \%$, respectivamente - , no entanto também foram apontadas reduções por meio das anomalias negativas identificadas no sudeste e principalmente no sul do Brasil, nas regiões do Atlântico Sudeste (RCP8.5, de 2006 a 2035), Atlântico Sul (RCP4.5, no período de 2066 a 2095) e no Uruguai (RCP4.5, de 2066 a 2095 e RCP8.5 no segundo e no terceiro período).

Para o cenário RCP4.5, a Figura 6 mostra que os modelos CanESM2 e CSIRO-Mk3 foram os mais pessimistas, estimando redução de até 2,76 mm/ano na maioria das regiões hidrográficas. O modelo CSIRO-Mk3 também se revelou pessimista no estudo de Guimarães et al. (2016), apontando o possível surgimento de uma extensa área hiperárida em algumas regiões do Ceará, no nordeste do Brasil. Para as regiões localizadas no sul do país - Atlântico Sul, Paraná e Uruguai - , os modelos não identificaram indícios de tendência significativa para a precipitação, com exceção da região hidrográfica do Uruguai, em que o modelo IPSL-CM5A-MR indicou tendência negativa de $1,48 \mathrm{~mm} / \mathrm{ano}$. Ainda sob esse cenário, o mesmo modelo sinalizou aumento da precipitação na maioria das regiões, especialmente no Atlântico Nordeste Ocidental, em que a tendência alcançou 7,66 mm/ano.
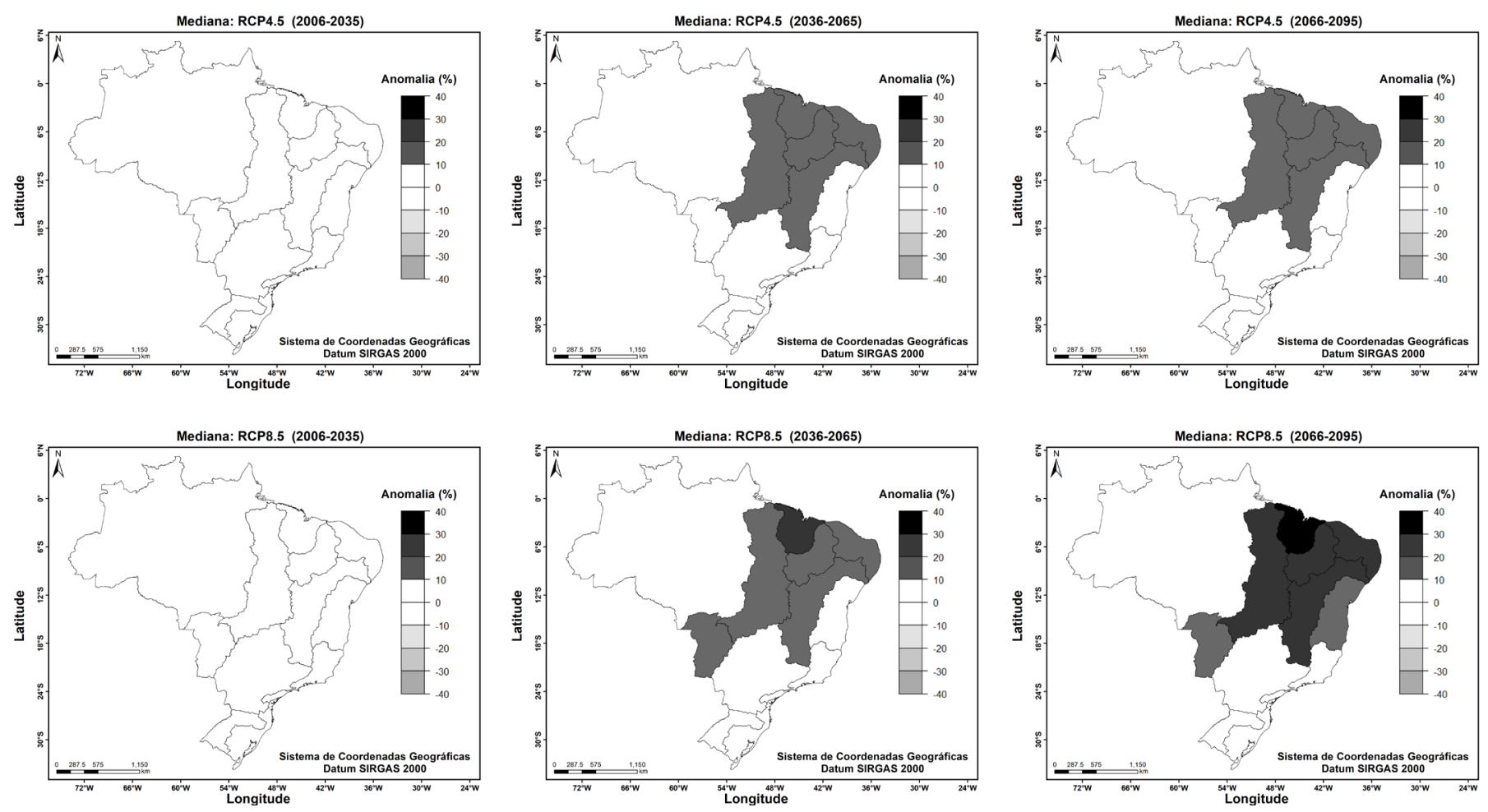

RCP: representative concentration pathways.

Figura 5 - Mediana das anomalias de precipitações anuais dos modelos do Coordinated Regional Climate Downscaling Experiment (CORDEX) para o cenário RCP4.5 no período de (A) 2006 a 2035; (B) 2036 a 2065 ; (C) 2066 a 2095; e para o cenário RCP8.5 no período de (D) 2006 a 2035; (E) 2036 a 2065; (F) 2066 a 2095. 
Para o cenário RCP8.5, no geral, identificou-se maior nível de incerteza associado à variável de precipitação, e a maioria dos modelos mostrou tendências significativas para grande parte das regiões hidrográficas e com grande dispersão dos seus módulos de declividade. As incertezas e dispersões encontradas podem estar relacionadas com o fato de as regiões associadas experimentarem variabilidade climática motivada por diversos sistemas atmosféricos e oceânicos, dificultando a representação climática da precipitação e um acordo entre os resultados apresentados pelos modelos (GUIMARÃES et al., 2016). Comparados ao cenário anterior, esses módulos foram bastante pronunciados, revelando uma possível inten- sificação no regime de precipitações anuais em grande parte das regiões hidrográficas, exceto as supracitadas localizadas no sul do país. Aqui, o Atlântico Sul foi a região com maior indicativo de ausência de tendência entre sete dos nove modelos. O modelo IPSL-CM5A-MR continuou atuando de forma otimista na maioria das regiões, estimando o aumento da precipitação em até $15,95 \mathrm{~mm} /$ ano, com destaque para o Atlântico Nordeste Ocidental. A redução mais significativa foi sinalizada para o Atlântico Leste, de 4,36 mm/ano pelo modelo CanESM2.

De acordo com o teste de Mann-Kendall-Sen realizado, todos os modelos do CORDEX apresentaram tendência positiva significativa para a variável temperatura e para

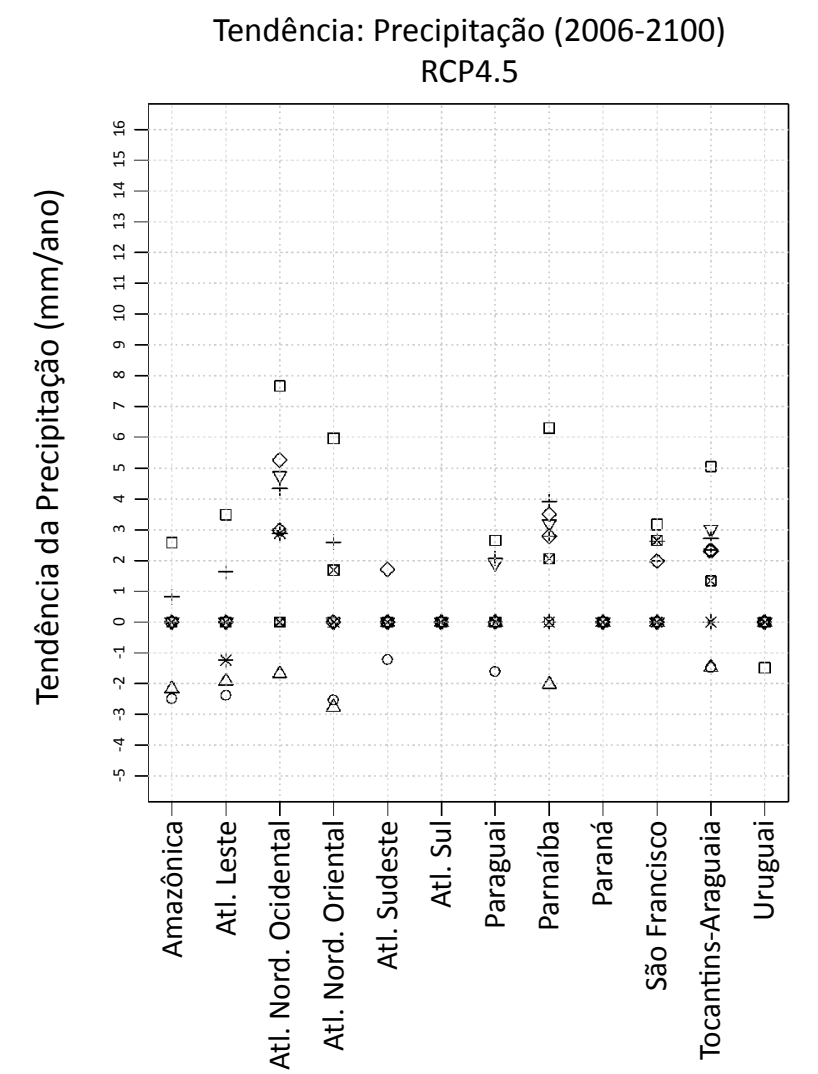

- CAnESM2 $\triangle$ CSIRO-Mk3 + EC-EARTH DIPSL-CM5A-MR $\diamond$ MIROC5
Tendência: Precipitação (2006-2100) RCP8.5

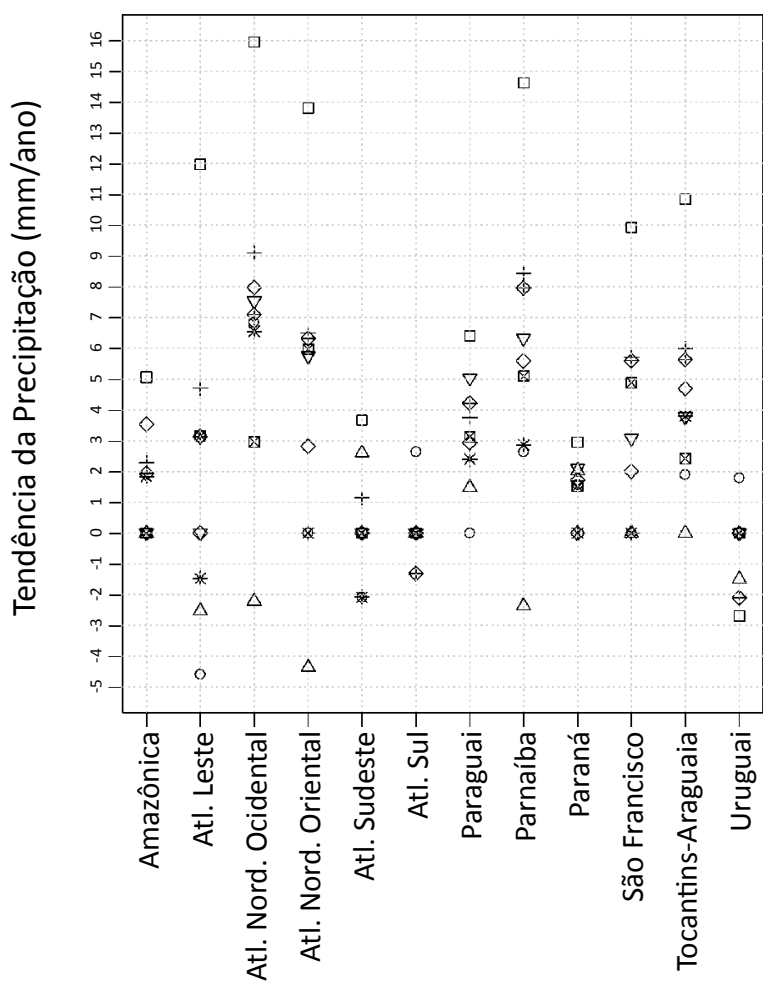

$\checkmark$ HadGEM2-ES $\otimes$ MPI-ESM-LR $\quad *$ NorESM1-M $\oplus$ GFDL-ESM2M

CanESM2: Canadian Earth System Model (The second generation); CSIRO-Mk3-6-0: Commonwealth Scientific and Industrial Research Organization (version Mk3-6-0); EC-EARTH: European Community-EARTH; IPSL-CM5A-MR: Institut Pierre Simon Laplace - 5 Component Models version A - Medium Resolution; MIROC5: Model for Interdisciplinary Research on Climate version 5; HadGEM2-ES: Hadley Center Global Environment Model version 2 - Earth System; MPI-ESM-LR: Max Planck Institute - Meteorology - Earth System Model Low Resolution; NorESM1-M: Norwegian Earth System Model version 1 - Medium resolution; GFDL-ESM2M: Geophysical Fluid Dynamics Laboratory Earth System Model version 2M; Atl.: Atlântico; Atl. Nord.: Atlântico Nordeste.

Figura 6 - Tendência da precipitação, segundo o teste de Mann-Kendall-Sen, para o período de 2006 a 2100 para os cenários representative concentration pathways (RCP) 4.5 e RCP8.5 nas regiões hidrográficas do Brasil. 
ambos os cenários em todas as regiões hidrográficas. Avaliando o cenário mais otimista RCP4.5, o conjunto de modelos indicaram aquecimento entre 0,12 e 0,59으 ano. O modelo CSIRO-Mk3, seguido de CanESM2 e IPSL-CM5A-MR, projetou as maiores tendências de aquecimento em todas as regiões, alcançando a marca de 0,59C/ano na RH Amazônica. O modelo GFDL-ESM2M projetou a menor tendência de temperatura na maioria das regiões, excetuando-se a do Uruguai, em que o modelo NorESM1-M foi responsável pelo menor incremento da variável. A menor tendência de aquecimento foi observada na região do Atlântico Sudeste. Tomando o cenário que destaca o aumento das emissões dos GEEs, RCP8.5, notaram-se tendências superiores ao cenário RCP4.5.

A Figura 7 mostra que os modelos CSIRO-Mk3 e IPSL-CM5A-MR indicaram tendências de aumento na temperatura média de até 1,0 e 0,95 ㅇano, respectivamente. A maior tendência de aquecimento, comparada à do cenário anterior, foi vista também na $\mathrm{RH}$ Amazônica: 1으/ano. Já o incremento na temperatura se demonstrou menos pronunciado na maioria das regiões hidrográficas pelos modelos NorESM1-M e GFDL-ESM2M, alcançando 0,38으ano para ambos. A menor tendência de aquecimento foi projetada pelo modelo MIROC5 para a RH Atlântico Sul: 0,35C/ano.

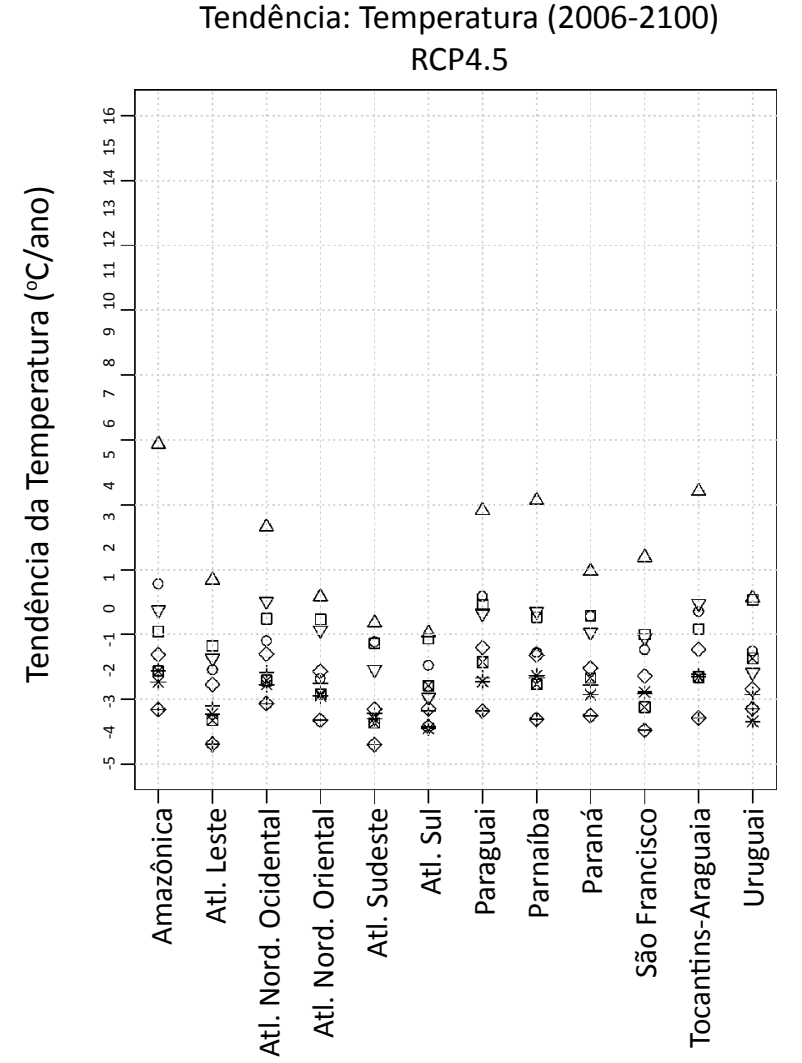

$\circ$ CanESM2 $\triangle$ CSIRO-Mk3 + EC-EARTH $\square$ IPSL-CM5A-MR $\diamond$ MIROC5
Tendência: Temperatura (2006-2100) RCP8.5

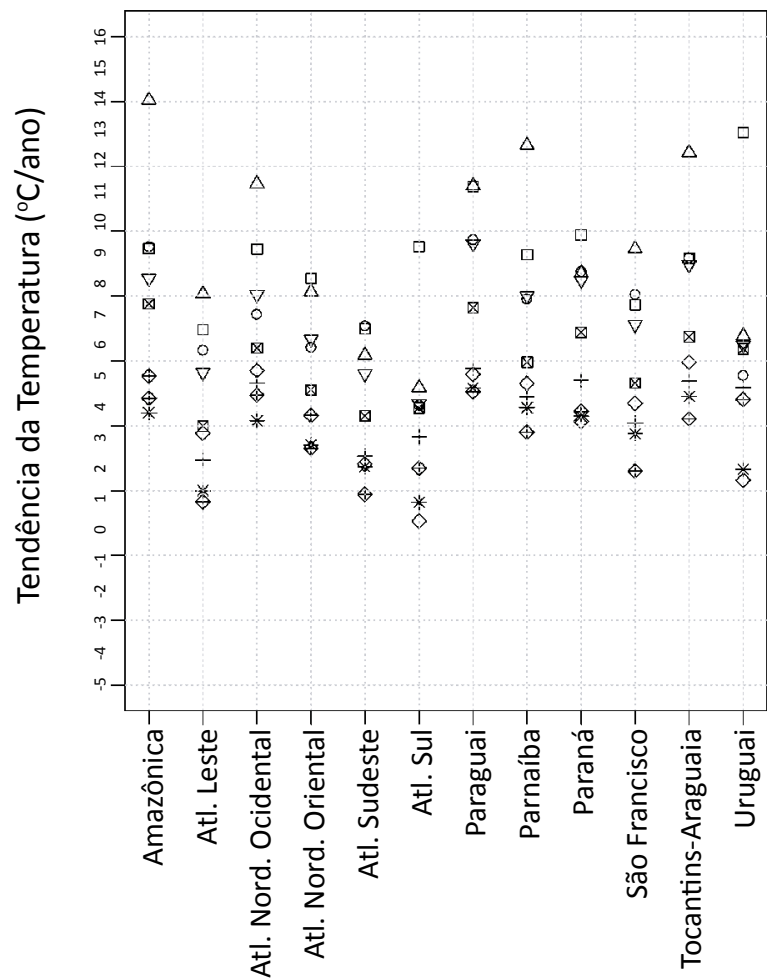

$\nabla$ HadGEM2-ES $₫$ MPI-ESM-LR * NorESM1-M $\oplus$ GFDL-ESM2M

CanESM2: Canadian Earth System Model (The second generation); CSIRO-Mk3-6-0: Commonwealth Scientific and Industrial Research Organization (version Mk3-6-0); EC-EARTH: European Community - EARTH; IPSL-CM5A-MR: Institut Pierre Simon Laplace - 5 Component Models version A - Medium Resolution; MIROC5: Model for Interdisciplinary Research on Climate version 5; HadGEM2-ES: Hadley Center Global Environment Model version 2 - Earth System; MPI- ESM- LR: Max Planck Institute - Meteorology - Earth System Model Low Resolution; NorESM1-M: Norwegian Earth System Model version 1 - Medium resolution; GFDL-ESM2M: Geophysical Fluid Dynamics Laboratory Earth System Model version 2M; Atl.: Atlântico; Atl. Nord.: Atlântico Nordeste.

Figura 7 - Tendência da temperatura, segundo o teste de Mann-Kendall-Sen, para o período de 2006 a 2100 para os cenários representative concentration pathways (RCP) 4.5 e RCP8.5 nas regiões hidrográficas do Brasil. 


\section{CONCLUSÕES}

O presente trabalho indica que a maioria dos modelos que compõem o projeto CORDEX divergiu quanto ao total precipitável observado em grande parte das regiões hidrográficas, como, por exemplo, as que abrangem o norte e o nordeste do Brasil, porém a climatologia averiguada foi razoavelmente bem representada no sul e sudeste do país, mesmo tendo sido subestimada. Os meses que se associam ao período chuvoso, em geral de novembro a abril, destacaram as maiores divergências entre as climatologias plotadas. O modelo CSIRO-Mk3 foi o que melhor representou a precipitação constatada, subestimando-a, no entanto.

Os modelos do CORDEX projetam o aumento da temperatura para as regiões estudadas, conforme resultados evidenciados nos trabalhos de Sales et al. (2015) e Guimarães et al. (2016). No período de 2006 a 2035, os cenários RCP4.5 e RCP8.5 mostraram-se semelhantes, permanecendo abaixo dos $0,5 \circ \mathrm{C}$ em todas as regiões. Levando em conta o cenário RCP8.5, as anomalias foram consideravelmente superiores nos períodos de 2036 a 2065 e de 2066 a 2095, em que os modelos indicaram valores entre 0,8 e $1,57^{\circ} \mathrm{C}$ em grande parte das regiões.

O conjunto de modelos indica que a precipitação também deve, em geral, aumentar para os dois cenários em algumas regiões hidrográficas. $O$ módulo das anomalias apresenta-se superior no cenário mais pessimista: RCP8.5. Nesse cenário, para o período de 2066-2095, a mediana sugeriu aumentos entre 10 e $30 \%$ nas RHs do Atlântico Leste, Atlântico Nordeste Ocidental, Atlântico Nordeste Oriental, Paraguai, Parnaíba, Tocantins-Araguaia e São Francisco. Anomalias negativas foram identificadas no sudeste e principalmente no sul do Brasil, como observado também no trabalho de Da Rocha (2014), nas regiões do Atlântico Sudeste (RCP8.5, de 2006 a 2035), Atlântico Sul (RCP4.5, no período de 2066 a 2095) e Uruguai (RCP4.5, de 2066 a 2095 e RCP8.5 no segundo e no terceiro período), sinalizando reduções na precipitação.

Avaliando individualmente os modelos de acordo com o teste de Mann-Kendall-Sen realizado, maior nível de incerteza associado à variável de precipitação foi observado para o cenário RCP8.5 quando comparado ao cenário otimista. A maioria dos modelos mostrou tendências significativas para grande parte das regiões hi- drográficas e com grande dispersão dos seus módulos de declividade, que, por sua vez, tiveram valores bastante pronunciados, sugerindo uma possível intensificação no regime de precipitações anuais em quase todas as regiões hidrográficas, exceto as do Atlântico Sul, Paraná e Uruguai. Em ambos os cenários, o teste apontou ausência de tendência na região do Atlântico Sul pela maioria dos modelos. O modelo IPSL-CM5A-MR foi o mais otimista e direcionado ao Atlântico Nordeste Ocidental. O modelo CanESM2, no entanto, apresentou-se como o mais pessimista, projetando a maior redução no Atlântico Leste no cenário RCP8.5.

Considerando a variável temperatura, todos os modelos apresentaram tendência positiva significativa e para ambos os cenários em todas as regiões hidrográficas. No cenário mais otimista, o modelo CSIRO-Mk3, seguido de CanESM2 e IPSL-CM5A-MR, projetou as maiores tendências de aquecimento, resultado que concorda com o trabalho de Guimarães et al. (2016). O modelo GFDL-ESM2M projetou a menor tendência de temperatura na maioria das regiões, excetuando-se a do Uruguai, em que o modelo NorESM1-M foi responsável pelo menor incremento da variável. A menor tendência de aquecimento foi observada na região do Atlântico Sudeste. Tendências superiores às do cenário RCP4.5 foram apresentadas pelos modelos CSIRO-Mk3 e IPSL-CM5A-MR (conforme também mostrado por Guimarães et al., 2016). A maior tendência de aquecimento foi verificada no norte do país, na RH Amazônica. Já o incremento na temperatura se demonstrou menos pronunciado pelos modelos NorESM1-M e GFDL-ESM2M na maioria das regiões hidrográficas. A menor tendência de aquecimento foi projetada pelo modelo MIROC5 para a RH Atlântico Sul.

Quanto ao nível de incerteza identificado com base em algumas divergências apresentadas nas projeções resultantes dos modelos do CORDEX, puderam-se traçar possíveis mudanças nos padrões de variabilidade dos campos de precipitação e temperatura das regiões hidrográficas do Brasil, o que, de acordo com o que foi sugerido por Silveira et al. (2018), permite auxiliar na adoção e no desenvolvimento de práticas de políticas e gestão de mitigação dos impactos provocados pelas mudanças climáticas sobre o nexo água-alimentoenergia no país. 


\section{AGRADECIMENTOS}

Os autores agradecem os apoios institucional e logístico oferecidos pela Coordenação de Aperfeiçoamento de Pessoal de Nível Superior - Código de Financiamento 001 - , pela Fundação Cearense de Apoio ao Desen- volvimento Científico e Tecnológico, pelo Conselho Nacional de Desenvolvimento Científico e Tecnológico e pelo Programa de Pós-Graduação em Engenharia Civil (Recursos Hídricos) da Universidade Federal do Ceará.

\section{REFERÊNCIAS}

AGÊNCIA NACIONAL DE ÁGUAS (ANA). Conjuntura dos recursos hídricos no Brasil: regiões hidrográficas brasileiras Edição Especial. Brasília: ANA, 2015. 163 p.

AGÊNCIA NACIONAL DE ÁGUAS (ANA). Levantamento da Cana-de-Açúcar Irrigada na Região Centro-Sul do Brasil. Brasília: ANA, 2017. 31 p.

AGÊNCIA NACIONAL DE ÁGUAS (ANA). Mudanças climáticas e recursos hídricos: avaliações e diretrizes para adaptação. Brasília: ANA, 2016. 93 p.

AGÊNCIA NACIONAL DO PETRÓLEO, GÁS NATURAL E BIOCOMBUSTÍVEIS (ANP). Biocombustíveis. Rio de Janeiro: ANP, 2017. Disponível em: <http://www.anp.gov.br/wwwanp/biocombustiveis?view=default>. Acesso em: 28 set. 2019.

AZEVEDO, A.N.G.; LIMA, B.G.A. Biocombustíveis: desenvolvimento e inserção internacional. Revista Direito Ambiental e Sociedade, Minas Gerais, v. 6, n. 1, p. 77-100, 2016.

BERGER, M.; PFISTER, S.; BACH, V.; FINKBEINER, M. Saving the Planet's Climate or Water Resources? The Trade-Off between Carbon and Water Footprints of European Biofuels. Sustainability, v. 7, n. 6, p. 6665-6683, 2015. http://doi. org/10.3390/su7066665

BOULAY, A.; BARE, J.; BENINI, L.; BERGER, M.; LATHUILLIÈRE, M.J.; MANZARDO, A.; MARGNI, M.; MOTOSHITA, M.; NÚÑEZ, M.; PASTOR, A.V.; RIDOUTT, B.; OKI, T.; WORBE, S.; PFISTER, S. The WULCA consensus characterization model for water scarcity footprints: assessing impacts of water consumption based on available water remaining (AWARE). The International Journal of Life Cycle Assessment, v. 23, p. 368-378, 2018. https://doi.org/10.1007/ s11367-017-1333-8

BRASIL. Ministério de Minas e Energia. Balanço Energético Nacional 2018. Brasília: MME/EPE, 2018. 62 p.

BURN, D.H.; ELNUR, M.A.H. Detection of hydrologic trends and variability. Journal of Hydrology, v. 255, n. 1-4, p. 107-122, 2002. https://doi.org/10.1016/S0022-1694(01)00514-5

CORREA, S.M.B.B. Probabilidade e Estatística. 2. ed. Belo Horizonte: PUC Minas Virtual, 2003. 116 p.

DA ROCHA, R.; REBOITA, M.S.; DUTRA, L.M.M.; LLOPART, M.; COPPOLA, E. Interannual variability associated with ENSO: present and future climate projections of RegCM4 for South America-CORDEX domain. Climatic Change, v. 125, n. 1, p. 95-109, 2014. https://doi.org/10.1007/s10584-014-1119-y

GIORGI, F.; JONES, C.; ASRAR, G. Addressing climate information needs at the regional level: The CORDEX framework. WMO Bulletin, v. 58, n. 3, p. 175-183, 2009.

GOPALAKRISHNAN, G.; NEGRI, M.C.; WANG, M.; WU, M.; SNYDER, S.W.; LAFRENIERE, L. Biofuels, land, and water: a systems approach to sustainability. US Department of Energy Publications, v. 35, 2009. Disponível em: <http:// digitalcommons.unl.edu/usdoepub/35>. Acesso em: 9 set. 2019. 
GUIMARÃES, S.O.; COSTA, A.A.; VASCONCELOS JÚNIOR, F.C.; SILVA, E.M.; SALES, D.C.; ARAÚJO JÚNIOR, L.M.; SOUZA, S.G. Projeções de Mudanças Climáticas sobre o Nordeste Brasileiro dos Modelos do CMIP5 e do CORDEX. Revista Brasileira de Meteorologia, São Paulo, v. 31, n. 3, p. 337-365, 2016. https://doi.org/10.1590/0102-778631320150150

MERCURE, J.F.; PAIM, M.A.; BOCQUILLON, P.; LINDNER, S.; SALAS, P.; MARTINELLI, P.; BERCHIN, I.I.; GUERRA, J.B.S.O.A.; DERANI, C.; ALBUQUERQUE JUNIOR, C.L.; RIBEIRO, J.M.P.; KNOBLOCH, F.; POLLITT, H.; EDWARDS, N.R.; HOLDEN, P.B.; FOLEY, A.; SCHAPHOFF, S.; FARACO, R.A.; VINUALES, J.E. System complexity and policy integration challenges: The Brazilian Energy- Water-Food Nexus. Renewable and Sustainable Energy Reviews, v. 105, p. 230-243, 2019. https:// doi.org/10.1016/j.rser.2019.01.045

MIRANDA, E. Potência agrícola e ambiental: áreas cultivadas no Brasil e no mundo. Agroanalysis, São Paulo, v. 38, n. 2, p. 25-27, fev. 2018.

MOREIRA, J.G.V.; NAGHETTINI, M. Detecção de Tendências Monotônicas Temporais e Relação com Erros dos Tipos I e II: Estudo de Caso em Séries de Precipitações Diárias Máximas Anuais do Estado do Acre. Revista Brasileira de Meteorologia, São Paulo, v. 31, n. 4, p. 394-402, set. 2016. https://doi.org/10.1590/0102-778631231420140155

NEW, M.; HULME, M.; JONES, P.D. Representing Twentieth-Century space-time climate variability. Part 1: Development of a 1961-1990 mean monthly terrestrial climatology. Journal of Climate, v. 12, p. 829-856, 1999. https://doi. org/10.1175/1520-0442(1999)012\%3C0829:RTCSTC\%3E2.0.CO;2

PRADO JÚNIOR, F.A.; ATHAYDE, S.; MOSSA, J.; BOHLMAN, S.; LEITE, F.; OLIVER-SMITH, A. How much is enough? An integrated examination of energy security, economic growth and climate change related to hydropower expansion in Brazil. Renewable and Sustainable Energy Reviews, v. 53, p. 1132-1136, 2016. https://doi.org/10.1016/j.rser.2015.09.050

REBOITA, M.S.; FERNANDEZ, J.P.R.; LLOPART, M.; ROCHA, R.P.; PAMPUCH, L.A.; CRUZ, F.T. Assessment of RegCM4.3 over the CORDEX South America domain: sensitivity analysis for physical parameterization schemes. Climate Research, v. 60, n. 3, p. 215-234, 2014. https://doi.org/10.3354/cr01239

ROTHAUSEN, S.G.S.A.; CONWAY, D. Greenhouse-gas emissions from energy use in the water sector. Nature Climate Change, v. 1, p. 210-219, 2011. https://doi.org/10.1038/nclimate1147

SALES, D.C.; COSTA, A.A.; SILVA, E.M.; VASCONCELOS JÚNIOR, F.C.; CAVALCANTE, A.M.B.; MEDEIROS, S.S.; MARIN, A.M.P.; GUIMARÃES, S.O.; ARAÚJO JUNIOR, L.M.; PEREIRA, J.M.R. Projeções de mudanças na precipitação e temperatura no Nordeste brasileiro utilizando a técnica de downscaling dinâmico. Revista Brasileira de Meteorologia, São Paulo, v. 30, n. 4, p. 435-456, 2015. http://dx.doi.org/10.1590/0102-778620140075

SCHNEIDER, U.; BECKER, A.; FINGER, P.; MEYER-CHRISTOFFER, A.; RUDOLF, B.; ZIESE, M. GPCC Full Data Reanalysis Version 6.0 at $0.5^{\circ}$ : Monthly Land-Surface Precipitation from Rain-Gauges built on GTS-based and Historic Data. 2011. http://dx.doi.org/10.5676/DWD_GPCC/FD_M_V7_050

SILVEIRA, C.S.; SOUZA FILHO, F.A.; LOPES, J.E.G.; BARBOSA, P.S.F.; TIEZZI, R.O. Análise das projeções de vazões nas bacias do setor elétrico brasileiro usando dados do IPCC-AR4 para o século XXI. Revista Brasileira de Recursos Hídricos, Porto Alegre, v. 19, n. 4, p. 59-71, out./dez. 2014. http://dx.doi.org/10.21168/rbrh.v19n4.p59-71

SILVEIRA, C.S.; SOUZA FILHO, F.A.; MARTINS, E.S.P.R.; OLIVEIRA, J.L.; COSTA, A.C.; NOBREGA, M.T.; SOUZA, S.A.; SILVA, R.F.V. Mudanças climáticas na bacia do rio São Francisco: Uma análise para precipitação e temperatura. Revista Brasileira de Recursos Hídricos, Porto Alegre, v. 21, n. 2, p. 416-428, jun. 2016. https://doi.org/10.21168/rbrh.v21n2.p416-428

SILVEIRA, C.S.; SOUZA FILHO, F.A.; VASCONCELOS JUNIOR, F.C.; ARAUJO JUNIOR, L.M.; CABRAL, S.L. Mudanças climáticas e o setor hidroelétrico brasileiro: uma análise com base em modelos do IPCC-AR5. Revista Brasileira de Ciências Ambientais, Rio de Janeiro, n. 47, p. 46-60, abr. 2018. https://doi.org/10.5327/Z2176-947820180210 
SOITO, J.L.S.; FREITAS, M.A.V. Amazon and the expansion of hydropower in Brazil: Vulnerability, impacts and possibilities for adaptation to global climate change. Renewable and Sustainable Energy Reviews, v. 15, n. 6, p. 3165-3177, 2011. https://doi.org/10.1016/j.rser.2011.04.006

SPIEGEL, M.R. Probabilidade e Estatística: Coleção Schaum. São Paulo: McGraw Hill do Brasil, 1978. 528 p.

STOCKER, T.; QIN, D.; PLATTNER, G.-K.; TIGNOR, M.; ALLEN, S.K.; BOSCHUNG, J.; NAUELS, A.; XIA, Y.; BEX, V.; MIDGLEY, P.M. Climate Change 2013: The Physical Science Basis. Contribution of Working Group I to the Fifth Assessment Report of the Intergovernmental Panel on Climate Change. Cambridge: Cambridge University Press, 2013. 1535 p.

TAO, H.; FRAEDRICH, K.; MENZ, C.; ZHAI, J. Trends in extreme temperature indices in the Poyang Lake Basin, China. Stochastic Environmental Research and Risk Assessment, v. 28, p. 1543-1553, 2014. https://doi.org/10.1007/s00477-014-0863-x

VAN VLIET, M.T.H.; FRANSSEN, W.H.P.; YEARSLEY, J.R.; LUDWIG, F.; HADDELAND, I.; LETTENMAIER, D.P.; KABAT, P. Global river discharge and water temperature under climate change. Global Environmental Change, v. 23, n. 2, p. 450-464, 2013. Global river discharge and water temperature under climate change. https://doi.org/10.1016/j.gloenvcha.2012.11.002

WAGESHO, N.; GOEL, N.K.; JAIN, M.K. Investigation of non-stationarity in hydro-climatic variables at Rift Valley lakes basin of Ethiopia, Journal of Hydrology, v. 444-445, p. 113-133, 2012. https://doi.org/10.1016/j.jhydrol.2012.04.011

YUE, S.; PILON, P.; CAVADIAS, G. Power of the Mann-Kendall and Spearman's rho tests for detecting monotonic trends in hydrological series. Journal of Hydrology, v. 259, n. 1-4, p. 254-271, mar. 2002. https://doi.org/10.1016/S00221694(01)00594-7 